\title{
LIBROS Y LECTURAS EN PONTEVEDRA EN 1871. UNA APROXIMACIÓN A SU ESTUDIO ${ }^{1}$
}

\section{BOOKS AND READINGS IN PONTEVEDRA IN 1871. AN APPROXIMATION TO HIS STUDY}

\author{
JUSTO CARNICERO MÉNDEZ-AGUIRRE
}

\begin{abstract}
Resumen
A lo largo del siglo XIX una explosión de editores e impresores, cubrirán el manto del territorio nacional. Darán a luz no sólo obras de producción propia de autores españoles, si no, que estos traducirán a los grandes extranjeros que por aquellos momentos estaban en boga (Chateaubriand, Sué, Ménégault, Richardson, Aimard, etc...). Autores como Eugenio de Ochoa, Rafael Mejía o mujeres como Joaquina García de Balmaseda o Ana María Poveda se encargarán de ello, para dar a conocer a los menos duchos en lenguas extranjeras estas obras literarias. Por otra parte, y no menos importante está la literatura gallega, Juan de Lérez, Benito Vicetto, Francisco Mirás, José Antonio dos Reis, o Saco y Arce, profesor en el Instituto de Pontevedra y autor de la Gramática Gallega. Gracias al catálogo de este bazar-ultramarinos e imprenta de José María Antúnez, del año 1871, conocemos el mercado del libro, las ediciones y novedades editoriales y los lectores que las adquirían, junto con el no menos interesante mercado importador de libros del extranjero queda reflejado en obras portuguesas y francesas, aunque eso sí, muy fragmentariamente.
\end{abstract}

\section{Palabras clave}

Edición, Siglo XIX, mercado del libro, lectores, lecturas, catálogo.

\begin{abstract}
Along the 19th century an explosion of publishers and printers, they will cover the mantle of the national territory. There will give birth not only works of own production of Spanish authors, if not, that these will translate into the big foreigners who for those moments were in vogue (Chateaubriand, Sué, Ménégault, Richardson, Aimard, etc ...). Authors like Eugenio de Ochoa, Rafael Mejía or women like Joaquina García de Balmaseda or Ana Maria Poveda will take charge of it, to announce the least expert in foreign languages these literary works. On the other hand, and not less importantly there is the Galician literature, Juan de Lérez, Benito Vicetto, Francisco Mirás, Jose Antonio two Reis, or Sack and Maple, teacher in the Institute of Pontevedra and author of the Galician Grammar. Thanks to the ultramarine catalogue of this bazaar and press of José María Antúnez, of the year 1871, we know the market of the book, the editions and publishing innovations and the readers who were acquiring them, together with the not less interesting importing market of books of the foreigner he remains reflected in Portuguese and French works, though it yes, very partially.
\end{abstract}

\section{Keywords}

Edition, 19th Century, Bought of the book, Readers, Readings, Catalogue

${ }^{1}$ Entregado el 21.12.2008. Las notas aquí referenciadas fueron tomadas del Fondo Casal del Museo de Pontevedra, signaturas y XLVIII/ A. 


\section{INTRODUCCIÓN.}

El siglo XIX, es para España un período convulso políticamente hablando y rural y atrasado, donde la incipiente industria radica en Madrid, Cataluña País Vasco y Valencia. Es en este período cuando haya unos inicios de educación y culturización de país con la Ley de 9 de septiembre de 1854, la llamada Ley Moyano, y la creación de una red nacional de institutos de Enseñanza Media. A medida que la sociedad va ganando en niveles de escritura y lectura se van afianzando crece progresivamente el número de librerías, imprentas y bibliotecas.

Es a comienzos de $\mathrm{S}$. XX cuando un país eminentemente rural se va a ir urbanizando vía emigración a los grandes núcleos de población, y con la llegada de estos nuevos habitantes su alfabetización progresiva a través de una incipiente red de escuelas e institutos.

Progresivamente, aparecen además de los libros como sistema de lectura, otros medios de distracción: cartillas, periódicos, juegos (La Oca), coplas, esquelas, etc... que para su comprensión es necesario tener al menos unos conocimientos básicos de leer.

El año 1871, es para España el comienzo de una nueva andadura de la primera República.

\section{METODOLOGÍA}

En el fondo Casal del Museo de Pontevedra se hallan un conjunto de catálogos de librerías de esta ciudad que muestran sus productos de venta cara al público. El primero de la librería de Antúnez y Pazos, C/Real 18, data de $1857 .{ }^{2}$ El segundo es de José A. Antúnez y Cía, 29 pp., y es de $1867 .^{3}$

El cuarto es de José Antúnez de 1887, sita la imprenta en la C/ del Puente, no $20^{4}$ y el quinto y último del año del desastre colonial, $1898 .{ }^{5}$

El tercer catálogo, el cual hemos reconstruido, junto con los anteriores y siguientes es el que hemos escogido para este pequeño estudio y su reconstrucción a través del Catálogo Colectivo del Patrimonio Bibliográfico Español, del que se indica el ${ }^{\circ}$ de asiento si está fuera de Galicia, y si es aquí se indica donde se halla el ejemplar.

El título va siempre en cursiva y es copia literal del citado catálogo.

${ }^{2}$ Museo de Pontevedra, Fondo Casal: 28/16. R. 19516.

${ }^{3}$ Museo de Pontevedra, Fondo Casal, 28/20.

${ }^{4}$ Museo de Pontevedra, Fondo Casal, 28/3.

${ }^{5}$ Museo de Pontevedra, Fondo Casal, 34/1. 
Cada ficha, reconstruida, lleva junto al autor, o traductor, en negrita, el título, lugar de edición, editor, data de la misma y paginación. En el área de notas en ocasiones reseñamos por una parte la colección donde fue publicada (veremos que son múltiples), y cuando fuera preciso el pseudónimo del autor/a de dicha obra.

Hemos creído oportuno localizar estos 103 títulos (en ocasiones con erratas tipográficas) que aparecen en el inventario y localizar los ejemplares conservados porque es la manera de ver, gracias a estos (la mayoría en la Biblioteca Nacional de Cataluña, Fondo Tusquets de Cabirol), lo que se adquiría y leía en Pontevedra en un año concreto, 1871. Y no sólo esto, si no más, conocer a sus poseedores, a través de los Fondos del Museo de Pontevedra y de la Biblioteca Pública del estado que celosamente guardan fondos de estos lectores. Serían algunos de estos: Víctor Said Armesto ${ }^{6}$, Andrés Muruais ${ }^{7}$, Jesús Murais ${ }^{8}$ y los médicos Rogelio Lois Estévez ${ }^{9}$ y José Casal Lois ${ }^{10}$, etc...

La importancia de las imprentas de la ciudad de Lérez, es que no sólo imprimirán libros, si no que además de ser tienda de ultramarinos y bazar (como veremos a continuación), ya en 1847 , la de D. José $\mathbf{M}^{\mathrm{a}}$ Vila aparece anunciada como: Imprenta, Librería y encuadernación”. ${ }^{11}$ Es decir realiza todo el proceso técnico de fabricación de un libro.

Respecto al oficio de impresor, se sigue con las sagas como en siglos anteriores, tal y como demostró D. Antonio Odriozola. Los Antúnez va su actividad desde 1860 hasta 1900 en que queda con la misma su viuda. ${ }^{12}$ Le preocupaba si funcionaba esta imprenta a Odriozola entre 1872 y 1880, y no localizó este catálogo, lo cual es la prueba de que sigue con su actividad en la Calle del Puente, $\mathrm{n}^{\mathbf{o}} 20$. En 1887 este mismo editor publicará su cuarto catálogo.

En la imprenta de Antúnez, además, verá la luz entre 1870 y 1871 el periódico El Derecho, formando parte de esa actividad editorial. ${ }^{13}$ Debemos recordar que en la ciudad de Pontevedra entre 1857, data del primer inventario que localizamos y 1898 se editan la nada despreciable cifra de 109 periódicos. ${ }^{14}$

\footnotetext{
${ }^{6}$ Los fondos se hallan en el Museo de Pontevedra. Véase COUCEIRO, A.: o. c., III, pp. 273-274.

${ }^{7}$ COUCEIRO, A.: o. c., II, p. 467. Sus fondos se hallan en la Biblioteca Pública de Pontevedra, fue médico.

${ }^{8}$ COUCEIRO, A.: $o$. c., II, p. 467. Fue Catedrático de Instituto.

${ }^{9}$ Su Biblioteca se halla en el Museo de Pontevedra. COUCEIRO, A.: o. c., II, p. 293

${ }^{10} \mathrm{Su}$ rica biblioteca de fondos del S. XIX se halla en el Museo de Pontevedra. A este legado corresponde este inventario. COUCEIRO, A.: o. c., II, pp. 293-294.

${ }^{11}$ ODRIOZOLA, A.: Las imprentas en Pontevedra en el S. XIX, p. 57

12 ODRIOZOLA, A.: o. c., pp. 109-115.

13 ODRIOZOLA, A.: o. c., p. 113

${ }^{14}$ ODRIOZOLA, A.: o. c., pp. 145-154
} 
Con respecto a los títulos, tal y como comprobará el lector, verá el aluvión de autores extranjeros que llegarán a Pontevedra sus traductores alguno como Eugenio de Ochoa de gran prestigio, junto con obras portuguesas y francesas sin traducir, lo que nos lleva a pensar que hay más de un conocedor de estas lenguas en la ciudad. Además los autores españoles de la época (Fernán Caballero, por ejemplo) o los locales (Juan de Lérez) no faltan en las estanterías de la librería junto a obras teatrales representadas en Madrid o en Pontevedra.

\section{EDICIÓN.}

[En portada orlada]

Imprenta, / Librería y EnCuadernación, / De/ D. José A. Antúnez y Cía./ [Filete]

CATÁlOgo general/ CORRESPONDIENTE AL AÑo DE/ 1871./

[Filete]

LIBROS DE INSTRUCCIÓN Y RECREO, / MENAJE PARA ESCUELAS, IMPRESOS/ PARA CORPORACIONES CIVILES Y MILI-/TARES ESCLESIÁSTICAS Y PARTICULARES/ CON VARIEDAD DE OBJETOS DE ESCRITORIO./

[Filete]

Pontevedra: Calle del Puente núm. 29

$40 \mathrm{pp}$.

Nos llama poderosamente la atención, que este establecimiento no sólo venda libros sino los más variopintos géneros tal y como se reseñan en los versos de la portada y contraportada.

En la primera aparece en siguiente descripción:

"En el mismo edificio y en local separado de los que ocupan la Imprenta, Librería y Encuadernación, se halla el establecimiento comercial de la propiedad exclusiva de Antúnez, que contiene los siguientes artículos:

AZÚCARES, almendra, avellanas, aceitunas legítimas de La Reina, aguardientes y licores, rom, aceite de rom, coñac, anisete, noyó y ginebra. Arroz, habichuela, almidón, azafrán en rama y molido y alpiste. Algodón, alpargatas, alfileres y abanicos ordinarios.

BREVAS e higos dulces de caja y serón y bacalao, barajas, bremantes (sic) y cuerdas de esparto y cáñamo.

Chocolates, cacaos y cascarilla, canela, café, cominos, pimienta y clavo, cacahuets verdes y tostados. Corchos, cola, cepillos ordinarios, caparrosa y caretas para máscaras. 
EFIGIES de pasta imitando mármol. Escobas y escobones.

FIDEO y diversidad de pastas. Fósforos.

GAS o petróleo refinado

JAMón tocino, chorizos y grasas. Jabón Málaga y Sevillano, id. de olor en pastillas.

LozA de porcelana: bacenillas, palanganas, jarros, jaboneras, cepilleras, fuentes, platos, pocillos, juegos de café, linternas económicas y floreros.

LOZA DE CRISTAL y vidrio: variedad de redomas, botellas de champagne, copas, vasos para agua, vino y licor, tacillas con platillo para té, id. id. y compotera para dulce, tarros para id., fanales, floreros, vasos de lámpara, embudos, escupideras, enjuagues, tubos para quinqué y tejas para invernáculos o claraboyas de $88 \mathrm{cms}$ de largo por 22 de ancho.

IDEM DE PEDERNAL de la fábrica “La Cartuja”: jaboneras, cepilleras, escupidores de orinal y chatos con mango, variedad de fuentes, platos, platillos, gícaras (sic)y pocillos, tarrones y tazas para caldo y café, jarros de barril con pico, lavamanos, orinales, id. chatos con mango para enfermos, pisteros para id., salseras de barco, id. soperitas. Tarrones de barro pintado para plantas. Librillos de fumar.

MARIPOSAS y morenillos

NUECES

Patatas y pimiento. Piedra-lumbre.

RESINA

$T E ́$

VINos del país y legítimos de Málaga añejo, de la hoja, lágrima, seco, moscatel, jerez y madera.

Velas de gasolina, esteáricas, cera y sebo.

YESCA

ZAPATONES y borceguíes.

Nos dan estas dos páginas una visión clara de lo que era un establecimiento de 1871 de la ciudad del Lérez.

El Catálogo en sí, también está divido como el anterior de 1867, por materias, indicándose en el mayor número de ocasiones el precio en reales del título indicado.

La primera sección la ocupa: "Menaje y otros efectos para escuelas"15 con variedad de surtidos, desde arenilla fina mineral a 7 cuartos la libra, barajas para jugar, colecciones de mapas, papel, etc..

El segundo apartado se titula: “Libros de religión, para la enseñanza elemental y superior, y de recreo" 16 .

\footnotetext{
526 Pp. 1-8

${ }^{527}$ Pp. 9-37
} 
Entre ellos destacamos:

"Aritmética escrita para escuelas rurales, por Don Juan Asúnsolo Martínez, catedrático especial de Naútica y Comercio de Rivadeo (sic) ................ sin precio ${ }^{17}$ Escuela de naútica y Comercio de Rivadeo [sic]. Asúnsolo Martínez, Juan.

Memoria leída por su Director Juan Asúnsolo Martínez: en la solemne apertura del curso académico de 1867 a 1868/ Escuela Especial de Naútica y Comercio de Rivadeo [sic].

Rivadeo: [s. n.], 1867, (Imprenta de Norberto Cascante).

17, [3] pp., [2] h. pleg.

CCPB: 000340408-0 ${ }^{18}$

Anuario de los progresos tecnológicos año $2^{\circ}$, tres tomos . ......................... $28^{19}$ Reales

Canalejas y Casas, José (1827-1902) ${ }^{20}$.

Anuario de progresos tecnológicos de la industria y de la agricultura / por D. José Canalejas y Casas.

Madrid: Carlos Bailly-Bailliere, 1863 (Imprenta de Bailly-Bailliere). CCPB: 000255692-8

La misma obra año $3^{\circ}$ tres tomos ..................... 28

Canalejas y Casas, José (1827-1902).

Anuario de los progresos tecnológicos de la industria y de la agricultura: año 1863/ por José Canalejas y Casas.

Madrid: Carlos Bailly-Bailliere, 1864, (Imprenta de Bailly-Bailliere).

464 pp.

CCPB: 000399152-0

\footnotetext{
${ }^{17}$ P. 9

${ }^{18}$ Santiago, Biblioteca Xeral Universitaria, Public. 1000.

${ }^{19}$ P. 10

${ }^{20}$ Ingeniero industrial y padre del político ferrolano José Canalejas Méndez. Revisó un Tratado de física experimental y aplicada y de meteorología con numerosa corrección de problemas/ por A. Ganot; traducido al castellano por José Molau, corregido por J. $M^{a}$. Pérez, $3^{a}$ ed. española, rev. y aum. según la última ed. francesa por José Canalejas y Casas. Madrid: Carlos Bailly-Bailliere, 1864.
} 
Agenda de la Lavandera ........................ 4

Poveda, María Ana, trad. ${ }^{21}$

Manual de las señoritas ó Arte de aprender cuantas habilidades constituyen el verdadero mérito de las mugeres [sic].../ traducido del francés por María Ana Poveda.

$2^{\text {a }}$ edición añadida con El Arte de La Lavandera y Lavado Doméstico.

Madrid: [s. n.], 1829 (Imprenta de Eusebio Aguado).

342, [80] pp.

CCPB: 000619903-8

Arte de hacer vino (enología) . . . . . . . . . . . . . . . . 6

Carbonell y Bravo, Francisco (1758-1837).

Arte de hacer y conservar el vino: con una noticia acerca de la fabricación del vinagre / por Francisco Carbonell y Bravo.

Barcelona: Antonio Brusi, 1820.

292 pp, [2] h. de lám.:il.

CCPB: 000485311-3

Adición al apéndice del arte de hacer y conservar el vino/ por Francisco Carbonell y Bravo.

Barcelona: Imprenta de la Viuda e Hijos de Brusi, 1824

16 pp., [3] h. de lám.: il.

CCPB: 000485318-0

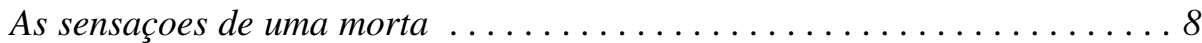

No localizada

Aventuras de Hércules Hardi, por Eugenio Sué . . . . . . . . . . . . . . . 4 Sué, Eugène (1804-1857).

Aventuras de Hércules Hardi ó La Guyana en 1772/ por Eugenio Sué; ilustrada con 12 grabados.

Madrid: [s. n.], 1855 (Imprenta del Semanario y de la Ilustración, a cargo de D.

G. Alhambra).

[2], 93 pp.:il.

Serie: Folletín de Las Novedades.

CCPB: 000177587-122

${ }^{21}$ No la cita Montesinos: o.c., en su índice de traductores.

22 Pontevedra, Biblioteca Pública, Fondo "Jesús Muruais", M. 962 (19) 
Antes de que te cases, mira lo que haces

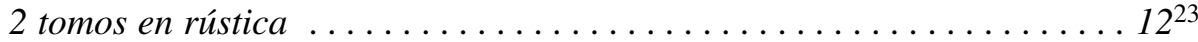

Kock, Charles Paul de (1793-1871).

Antes de que te cases mira lo que haces/ Obras de Ch. Paul de Kock.

Madrid: Librería e imprenta de D. León Pablo Villaverde, 1865

2 v. (T. I. 213 pp.- T. II. 245 pp.)

Serie: Biblioteca Enciclopédica. Sección segunda. Lectura amena y Festiva; I-II.

CCPB: 000756667-0

Amelia Both 5 tomos en pasta ......................... 20

Fielding, Henry (1707-1754). ${ }^{24}$

D. A. R. G. trad.

Historia de Amelia Booth/ escrita por el famoso Fielding; traducida al castellano por D. R. A. G. Tomo I.

Madrid: en la Imprenta de la Viuda de Ibarra, 1795.

[8], $336 \mathrm{pp}$.

CCPB: 000057299-3

Historia de Amelia Booth /.... Tomo segundo.

Madrid: En la imprenta de la Viuda de Ibarra, 1795.

[10], $276 \mathrm{pp}$.

CCPB: 000057300-025

Historia de Amelia Booth/... Tomo tercero.

Madrid: En la imprenta de la Viuda de Ibarra, 1796.

[8], $370 \mathrm{pp}$.

CCPB: 0000057301-926

Historia de Amelia Booth /... Tomo cuarto.

Madrid: en al Imprenta de la Viuda de Ibarra, 1796.

[2], $250 \mathrm{pp}$.

CCPB: 000057302-727

Arte de hacerse amar por su marido, por D. Miguel Herrero, rústica . . . . 40 Blanco Herrero, Miguel.

Arte de hacerse amar por su marido/ por Miguel Blanco Herrero.

23 P. 11

${ }^{24}$ MONTESINOS, J. F.: o. c., p. 193

${ }^{25}$ Se conserva este tomo en A Coruña, Biblioteca del Consulado, S 3 F; 1-11; 358

${ }^{26}$ A Coruña, Biblioteca del Consulado, S 3 F; 1-11, 359

${ }^{27}$ A Coruña, Biblioteca del Consulado, S 3 F; 1-11; 360. 
Madrid: [s. n.], 1869 (Imprenta de la Biblioteca Universal Económica). $368 \mathrm{pp}$.

CCPB: 000101719-5

Boletín bibliográfico de 1842, 1843, 1845, 1846 y 1849 a . . 6 rs cada tomo Boletín bibliográfico españoly estrangero [sic]: compremde [sic] todas las publicaciones nuevas españolas, de obras, folletos, periódicos, grabados, litografías, cartas geográficas, etc... muchas de las publicadas en los años pasados, y las más principales hechas en el estrangero [sic]...

Madrid: Librería Europea, 1842.

V. (T. III. [4], 430 pp.)

Se publica el $1^{\circ}$ y el 16 de cada mes, en cuadernos de 16 páginas, todos los números forman un tomo con su correspondiente índice ó tabla general clasificada por órden de materias y autores.

CCPB: 000768761-3

No localizados los de los años 1843, 1845, 1846 y 1849.

Breve y verdadera historia del maestrazno $2^{28}$

\section{Galdo López de Neira, Manuel Ma . José (1825-1895)}

Breve historia del mastranzo: planta preconizada como uno de los mejores específicos para combatir el cólera-morbo/ por Manuel María José de Galdo.

Madrid:[s. n.], 1855 (Imprenta de Higinio Reneses).

$23 \mathrm{pp}$.

CCPB: 000248478-129

\section{Galdo López de Neira, Manuel Ma . José (1825-1895)}

Breve historia del mastranzo: planta preconizada como uno de los mejores específicos para combatir el cólera-morbo/ por Manuel María José de Galdo.

Ed. rev. y corr.

Madrid: [s. n.], 1856 (Imprenta de Manuel Galiano).

$27 \mathrm{pp}$.

CCPB: 000129378-8 30

\footnotetext{
${ }^{28}$ P. 12 del inventario.

${ }^{29}$ Ejemplar en la Biblioteca Pública de Pontevedra, V. 43-16 con dedicatoria manuscrita del autor.

${ }^{30}$ Hay ejemplar en la Biblioteca Pública de Pontevedra, V. 13-15, con dedicatoria manuscrita del autor. La única biblioteca española, que posee las dos ediciones.
} 
Cuaderno de lectura primero que sirvió para

la enseñanza del ex-príncipe Alfonso de Borbón ${ }^{31} \ldots \ldots \ldots \ldots \ldots \ldots . \ldots$

Merino Ballesteros, Francisco.

Merino Ballesteros, Ramón.

Cuaderno primero para enseñar a leer a S. A. R. el Serenísimo Señor Príncipe de Asturias / compuesto por F. y R. Merino Ballesteros.

$3^{\mathrm{a}}$ ed.

Madrid: [s. n.], 1863 (Imprenta de Anoz).

64 pp.,[1] h. de lám.

CCPB:000470114-3

(...)

Clara Harlowe, 9 tomos desde el $2^{\circ} \ldots \ldots \ldots \ldots \ldots \ldots \ldots \ldots \ldots . \ldots 40$

Richardson, Samuel (1689-1761).

Le Tourneur, M. trad.

Gutiérrez, José Marcos, trad. ${ }^{32}$

Clara Harlowe: novela/ traducida del inglés al francés por Mr. Le Tourneur, siguiendo en todo la edición original revista por su autor Richardson, y del francés al castellano por José Marcos Gutiérrez.

$2^{a}$ ed. corr. y enmendada

Madrid: [s. n.], 1829 (Imprenta que fue de Fuentenebro).

9 v. (T. I. XXII, 412pp.--T. VI. 348 pp.)

CCPB: 000681794-7

Confesión general por Sulié, 4 tomos .................... 20

Soulié, Frédéric (1800-1847). ${ }^{33}$

Confesión general / por Federico Soulié; traducida al castellano.

Madrid: [s. n.], 1849 (Impr. de Gabriel Gil).

7 v. (T. I. 215 pp.-T. II. 215 pp.- -. T. III. 214 pp.-T. IV. 215 pp. -.T. V. 222

pp. T. VI. 220 pp. -.T. VII. 302 pp.)

CCPB: 000679271-5

${ }^{31}$ Cuando se hace el catálogo está la I ${ }^{\mathrm{a}}$ República.

32 MONTESINOS, J. F. : o. c., p. 233

${ }^{33}$ MONTESINOS, J. F.: $o$. c., pp. 246-248 
Corazón leal, por Gustavo Aimard . . . . . . . . . . . . . . . 8

Aimard, Gustave (1818-1883).

Sáenz de Urraca, J. F., trad. ${ }^{34}$

Corazón leal: novela escrita en francés/ por Gustave Aimard; traducción de J. F.

Sáenz de Urraca.

Madrid: Bailly-Baillière, 1865.

446 pp.

Gustave Aimard es el pseudónimo de Olivier Gloux.

CCPB: 000084644-9

Creencias y desengaños por Navarrete . . . . . . . . . . . . . . . 8

Navarrete y Fernández-Landa, Ramón de (1818-1897) ${ }^{35}$.

Creencias y desengaños/ por Ramón de Navarrete.

Madrid: [s. n.], 1843 (Imprenta de la Viuda de Jordán e hijos).

$211 \mathrm{pp}$.

CCPB: 000540200-X

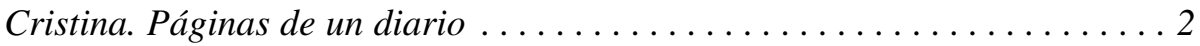

Vicetto Pérez, Benito (1824-1878) ${ }^{36}$.

Cristina: pájinas [sic] de un diario / por Benito Vicetto.

Sevilla: [s. n.], 1852 (Imprenta de Gómez y Oro).

2 v. (T. I. 177 pp.-T. II. 178 pp.)

CCPB: 000687581-5

Crímenes célebres ............................8

Palomera y Ferre, Carlos Nicolás de.

Dramas sangrientos, o colección de completa de los más célebres crímenes de todos los países/ por Carlos Palomera y Ferrer.

Madrid: Jesús Gracia, 1867-1868.

3 v. :il

CCPB: 000169890- $7^{37}$

\footnotetext{
${ }^{34}$ No lo cita Montesinos.

${ }^{35}$ FERRERAS, J. I.: $o$. c., pap. 1393

${ }^{36}$ Nacido en Ferrol, fue uno de los precursores del "Rexurdimento" en Galicia, publicó en 1865 la "Historia de Galicia". COUCEIRO, A.: o. c., III, pp. 499-501.

${ }^{37}$ Hay dos tomos en la Biblioteca Pública de Pontevedra, T. I., 930 pp.-T. II.112 pp., con signatura R. 458. En el Museo de esta ciudad hay otro con signatura FV.12058
} 
Calendario de Valladolid de $1871 \ldots \ldots \ldots \ldots \ldots \ldots$ sin precio ${ }^{38}$ No localizada

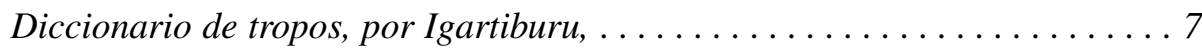

Igartuburu, Luis de.

Diccionario de tropos y figuras de retórica: con ejemplos de Cervantes/ por Luis de Igartuburu.

Madrid: [s. n.], 1842 (Imprenta de Alegría y Clarín).

XIV, $257 \mathrm{pp}$.

CCPB: 000202091-2

Diccionario Italiano-español y español-italiano por D. Roque Barcia ................................. 24

\section{Caccia, Guiseppe}

Nuevo diccionario italiano-español y español-italiano: con la pronunciación figurada en ambas lenguas / por José Caccia...

París: Librería Garnier Hermanos, 1869.

$\mathrm{XV}, 528,333 \mathrm{pp}$.

CCPB: 000266571-9

Diccionario Latino-español aumentado con más de

20.000 voces por Valvuena ............................ 64

Valbuena, Manuel de.

Martínez López, Pedro, ed. lit.

Valbuena reformado Diccionario latino-español aumentado con más de 20000 voces...: lleva además un vocabulario español-latino/edición hecha bajo la dirección de M. D. P. Martínez López.

Madrid: En la Librería de C. Bailly-Baillière, 1851.

XI, 954, 235 pp.

Texto a tres columnas

CCPB: 000308320-9

Idem. Id. por Gimenez

18

Giménez, Esteban.

Martín de Heredia, Antonio, corr. y aum.

38 P. 15 del inventario. 
Diccionario manual latino-español: dispuesto para uso de los jóvenes que estudian la lengua latina/ por Esteban Giménez; corregido y aumentado en esta nueva impresión con la parte mitológica y otras voces... por Antonio Martínez Heredia.

Madrid: Juan Díaz de Los Ríos, 1834.

764, [79] pp.

CCPB: 000220691-9

Diccionario latino-español etimológico, por

D. Raimundo de Miguel y el Marqués de Morante ................80

Miguel y Navas, Raimundo de (1816-1878).

Morante, Joaquín Gómez de la Cortina, Marqués de (1808-1868).

Nuevo diccionario latino-español etimológico: escrito con presencia de las obras más notables...: seguido de un tratado de sinónimos y de un vocabulario español-latino/ por Raimundo de Miguel y el Marqués de Morante.

$3^{\text {a }}$ ed. corr. y aum.

Madrid: Agustín Jubera, 1871 (Imprenta de Manuel Minuesa).

XXXIX, 997, 76, 256 pp.

CCPB: 000450528-X

Diccionario de bibliografía agronómica de toda clase de

escritos relacionados con la agricultura; seguidos de

un índice de autores y traductores por D. Braulio

Antón Ramírez, 1 tomo abultado ......................66 . . . . . .

\section{Antón Ramírez, Braulio (1823-1892).}

Diccionario de bibliografía agronómica y de toda clase de escritos relacionados con la agricultura, seguido de un índice de autores y traductores con algunos apuntes bibliográficos / su autor Braulio Antón Ramírez.

Madrid: [s. n.], 1865 (Imprenta y estereotipia de M. Rivadeneyra).

XVIII, 1015 pp.

Obra premiada por la Biblioteca Nacional en concurso público de 5 de enero de 1862.

CCPB: 0000086725- $\mathrm{X}^{40}$

39 P. 16

40 Se encuentran tres ejemplares en Galicia. Lugo (Biblioteca Pública, $\mathrm{n}^{\circ}$ 7792) y Pontevedra (B. Pública, R/2215) y Museo (FV-12157) 
Diego Tinoco ou a Corte de D. Joâo $2^{\circ}$ en 1484.

drama en tres actos . . . . . . . . . . . . . . . . . . . . . . ...4

J. P. M. S.

Diogo Tinoco ou a Côrte de D. Joâo II: drama/ por J. P. M. S.

Porto: Tip. Comercial, 1840.

56 pp.

Lisboa, Biblioteca Nacional L.25553/26 y L. 46854

Danzas en litografía para cantar por música

los días de Carnaval ............................. $6^{41}$

No localizada

El Parnaso de los niños

.4

Caballero de Rodas, $\mathbf{C}$.

El Parnaso de los niños: o sea colección de poesías en diferentes rimas de los mejores poetas españoles. Comprende además un número poético de la Historia de España/ por C. [Caballero] de Rodas...; lo publica la Casa de Educación de D. José González.

Madrid: [s. n.], 1867 (Imprenta de Manuel Minuesa).

Madrid, Biblioteca Nacional (J 2 C/226/2)

El buen Sancho de España ................. no tiene precio. El buen Sancho de España: colección metódica de máximas, proverbios, sentencias, y refranes acerca de la agricultura, la ganadería y la economía rural/ escritos y anotados por un espíritu apasionado de las gentes del campo.

Madrid: s. n.], 1862 (Librería de León Pablo Villaverde).

$128 \mathrm{pp}$.

CCPB: 000753895-2

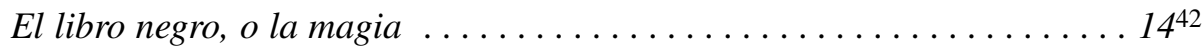

Flamel, Hortensio.

El libro negro ó La Magia: las ciencias ocultas, la alquimia y astrología, con secretos y recetas admirables, sacados de los más célebres autores cabalísticos tanto antiguos como modernos: el arte de adivinar por los naipes y una nueva llave de sueños / por Hortensio Flamel.

\footnotetext{
${ }^{41}$ p. 17

${ }^{42}$ P. 18
} 
$4^{\mathrm{a}}$ ed.

Barcelona: [s. n.],1860 (Imprenta de Manuel Saurí)

254 pp.:il.

CCPB: 000465001-8

Escritura del Patronato de Figueroa . $4^{43}$

Patronato laical Manuel Ventura Figueroa.

Escritura de fundación del Patronato laycal y memorias del Excmo. Sr. D. Manuel Ventura de Figueroa: otorgada por sus testamentarios a consequencia del poder y facultad que para ello dexó en su testamento y últimas disposiciones.

Madrid: En la Imprenta de Joachin Ibarra, 1784.

$42 \mathrm{pp}$.

Santiago, Biblioteca Universitaria (Foll. Carp. 6-2)

El Fuero Viejo de Castilla 10

Asso y del Río de (1742-1814), Ignacio Jordán de, anot.

Manuel y Rodríguez, Miguel de, anot.

El Fuero Viejo de Castilla: sacado, anotado y comprobado con el ejemplar de la misma obra, que existe en la Real Biblioteca de esta Corte, y con otros dos mss./ publícanlo con notas históricas y legales los doctores Ignacio Jordán de Asso y del Río y Miguel de Manuel y Rodríguez.

Madrid: Librerías de los Señores Viuda e Hijos de Antonio Calleja y de Manuel Pereda; Lima: casa de los Señores de Calleja, Ojea y Compañía, 1847 (Imprenta de Alejandro Gómez Fuentenebro).

XLVI, 139 pp.

CCPB: 000194758-3

El cultivo mejorado de la vid en España, arreglado

al clima de Vega y Ortiz . . . . . . . . . . . . . . . . . . . . . 6

\section{Vega y Ortiz, Domingo de la}

El cultivo mejorado de la vid/ por Domingo de la Vega y Ortiz.

Madrid: [s. n.], 1863 (Imprenta de las Novedades, a cargo de J. Trujillo).

172 pp.

Biblioteca Agrícola de las Novedades.

CCPB: 000666276-5

43 P. 19 
Elena de Mendoza, novela original de la señorita

Dña. Francisca Carlota del Riego, precedida de un

prólogo de D. Vicente Barrantes ........................ 24

Riego Pica, Francisca Carlota de ${ }^{44}$

Barrantes, Vicente (1829-1898), prolog.

Elena de Mendoza / novela original de Francisca Carlota del Riego Pica; precedida de un prólogo de Vicente Barrantes.

Madrid: [s. n.], 1859 (Impr. de J. Antonio Ortigosa)

XIV, 380 pp., [4] h. de lám.:il.

CCPB: 000679328-2

El romancero de los once Alfonsos . .................... 6

Velasco Ayllón, Ricardo (n. 1859).

Fuentes, Eduardo

El romancero de los once Alfonsos, Reyes de Asturias, León y Castilla/ por Ricardo Velasco Ayllón y Eduardo Fuentes.

Madrid: Librería Americana, 1863, (Imprenta de A. Sta. Coloma)

$147 \mathrm{pp}$.

CCPB: 000387054-5

Enrique de Valmore 2 tomos ........................ 10

Ménégault, A. P. F. (1770- post. 1830). ${ }^{45}$

Collado, José del, trad.

Enrique de Valmore/ escrita en francés por $\mathbf{M}^{* * * * ;}$; publícale en castellano José del Collado.

Madrid: [s. n.], 1821 (Imprenta del editor).

2 v. (T. I. 247 pp., [1] h. de lám.- - T. II. 259 pp., [1] h. de lám.:il.)

$\mathrm{M}^{* * * * *}$ es un pseudónimo de A. P. F. Ménégault.

CCPB: 000675654-9

El Palacio de Saintdizier.- La jesuita ...................... 2

No localizada.

${ }^{44}$ FERRERAS, J. I.: $o$. c., pap. 1703.

${ }^{45}$ MONTESINOS, J. F.: $o$. c., pp. 223-224 
Ensayo sobre las revoluciones de Chateaubriand ............... 16

Chateaubriand, François-René, vicomte de (1768-1848)..$^{46}$

Madina-Veytia, Francisco, trad.

Ensayo sobre las revoluciones antiguas / por F. A. Chateaubriand; traducida por Francisco Madina-Veytia.

Madrid: Imprenta de Gaspar y Roig, editores, 1856

155 pp.:il.

Biblioteca ilustrada Gaspar y Roig.

CCPB:000194313-8 $8^{47}$

El renegado tres tomos . ........................... 10

Arlincourt, Charles-Victor Prévost d'(1788-1856). ${ }^{48}$

El Renegado/ por el V. de Arlincourt; traducido de la última edición francesa. $2^{\mathrm{a}}$ ed.

Barcelona: Imprenta y Librería de E. Pujal, 1849.

3 v. (T. I. 237 pp., [1] h. de lám. - T. II 237 pp., [1] h. de lám. - T. III. 223 pp., [1] h. de lám.:il)

CCPB: 000674580-6

El filósofo por amor $\ldots \ldots \ldots \ldots \ldots \ldots \ldots \ldots \ldots \ldots \ldots \ldots$

No localizada.

Elena Virgínea ................................ 12

Richardson, Samuel (1689-1761). ${ }^{49}$

Elena virginia, o Historia de una joven rusa / [Samuel Richarson].

Valencia: por Domingo y Mompié, 1818-1819.

3 v. (T. I. [8], 219 pp.-T. II. 291 pp. .— T. III. 267 pp., [3] h. de lám.: il.)

CCPB:000555165-X

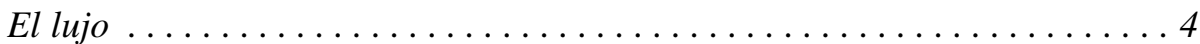

Grassi de Cuenca, Ángela (1826-1883). ${ }^{50}$

\footnotetext{
46 MONTESINOS, J. F.: $o$. $c$., pp. 171-175

${ }^{47}$ En Galicia hay ejemplares de esta edición en Lugo, Biblioteca Pública, signatura 5452 (4) y en Pontevedra, Biblioteca Pública, (R-540) y Museo (V.S.A. 16119, Fondo Víctor Said Armesto y FV. 11746(6), Fondo Filgueira Valverde).

${ }^{48}$ MONTESINOS, J. F.: $o$. c., pp. 155-156

${ }^{49}$ MONTESINOS, J. F.: $o . c$., p. 233.

${ }^{50}$ FERRERAS, J. I.: o. c., pap. 916
} 
El lujo: novela de costumbres/ original de Ángela Grassi.

Madrid: [s. n.], 1865 (Imprenta de la Academia Tipográfica).

$261 \mathrm{pp}$.

Serie: Biblioteca del Hogar

CCPB: 000616789-6

El Gil-Blas de la revolución, 2 tomos

10

Picard, Luois-Benoît (1769-1828).

\section{A.B. Trad.}

El Gil Blas de la Revolución ó confesiones de Lorenzo Giffard/ por L. B. Picard, traducido de la segunda edición por A. B.

Barcelona: [s. n.], 1838 (Imprenta de Antonio Bergnes).

265 pp.

Serie: Biblioteca selecta y económica. Sección $1^{\mathrm{a}}, \mathrm{n}^{\mathrm{o}} 2$.

CCPB: 000290492-6

El que nace para ochavo. apuntes de nuestra hacienda,

por un Ministro del ra...bo . . . . . . . . . . . . . . . . . . . . 4 $4^{51}$

El que nace para ochavo... apuntes de nuestra Hacienda, por un ministro del ra..bo.

Madrid: [s. n.], 1869 (Imprenta de R. Labajos).

$88 \mathrm{p}$.

CCPB: 000330890-1

Fabulas de Fedro, pergamino ...................

Fedro, Gayo Julio (15 a.C.- 55 d. C.).

Cepeda, Francisco de, corr.

Fábulas de Fedro, Liberto de Augusto/ ilustradas con algunas notas más de las que tenían para la fácil inteligencia y uso de los principinates en las escuelas de gramática; y corregidas con mayor exactitud por D. Francisco de Cepeda...

Madrid: [s. ,], 1851 (Imprenta de D. Julián Viana).

222 pp.

CCPB: 000629355- $7^{52}$

\footnotetext{
51 P. 20

52 Hemos localizado dos ejemplares encuadernados en pergamino, uno en la Abadía de Sto. Domingo de Silos (BL4-g 146) y el otro en Azpeitia, Santuario de Loyola (3086/1-40)
} 
Fabulas de Samaniego 3

Samaniego, Félix María de (1745-1801).

Fábulas en verso castellano para uso de las escuelas/ compuestas por Félix María de Samaniego.

La Coruña: [s. n.], 1824 (Imprenta de Rodríguez y Cía).

2 v. (T. I. 193 pp.)

CCPB: 000162165-3 $3^{53}$

Fisiología del sastre $\ldots \ldots \ldots \ldots \ldots \ldots \ldots \ldots \ldots \ldots \ldots \ldots \ldots$

Huart, Louis (1813-1865). ${ }^{54}$

M. M., trad.

Fisiolojía [sic] del sastre/ por Louis Huart; con dibujos de Gabarri; traducida al castellano por N. N.

Barcelona: [s. n.], 1848 (Imprenta de Juan Oliveras).

112 pp.:il.

Madrid, Biblioteca Nacional (12/248075)

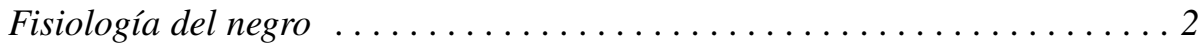

J. M. de A., trad. ${ }^{55}$

Fisiología del negro/ traducción libre por J. M. de A.

Madrid: Roix, editor, 1842.

141 pp., [4] h. de lám.:il.

CCPB:000284976-3

Gramática hispano-latina por D. Raimundo de Miguel,

$9^{a}$ edición

\section{Miguel y Navas, Raimundo de (1816-1878).}

Gramática hispano-latina teórico-práctica: para el estudio simultáneo de las lenguas latina y castellana comparadas/ escrita por Raimundo de Miguel.

$9^{\mathrm{a}}$ ed.

Madrid: Agustín Jubera, 1867.

299 pp.

CCPB: 000167698-9

\footnotetext{
53 A Coruña, Real Academia Galega, signatura 17098, tan sólo el T. I.

${ }^{54}$ MONTESINOS, J. F.: o. c., p. 228

55 MONTESINOS, J. F.: o. c., p. 285
} 
Gramática gallega ............................ $15^{56}$ Mirás, Francisco. ${ }^{57}$

Compendio de gramática gallega-castellana: con un vocabulario de nombres y verbos gallegos y su correspondencia castellana....; un grandioso poema de 100 octavas titulado la Creación y La Redención; un extracto de fábulas...algunas del autor.../ por Francisco Mirás...

Santiago: [s. n.], 1864 (Establecimiento Tipográfico de Manuel Mirás).

147 pp., [1]h

CCPB: 000168286-5 58

Genio y Figura hasta la sepultura ...................... 14

Valera, Juan (1824-1905). ${ }^{59}$

Genio y figura/ Juan Valera

Madrid: [. n., s. a.] (Imprenta Alemana).

$271 \mathrm{pp}$.

Serie: Obras completas de Juan Valera. Novelas, $\mathrm{n}^{\circ} 10$.

CCPB: 000356467-3

Galería de españoles célebres contemporáneos

o biografías y retratos por Nicomedes Pastor Díaz .............. 2

Pastor Díaz, Nicomedes (1811-1863). ${ }^{60}$

Cárdenas, Francisco de

Galería de españoles célebres contemporáneos, ó Biografías y retratos de todos los personages [sic] distinguidos de nuestros días en las ciencias, en la política, en las armas, en las letras y en las artes/ publicadas por Nicomedes Pastor Díaz y Francisco de Cárdenas.

Madrid: [s. n.], 1842 (Imprenta de Vicente Lalanda).

53, 64, 53, 71, 39, 31 pp., [4] h. de lám. il.

Contiene: Manuel Bretón de los Herreros, Nicolás María Garelly, Antonio Gil y Zárate, Francisco Javier de Buros, Alejandro Aguado y Juan Bravo Murillo.

Cada sección de página corresponde a un biografiado.

CCPB: 000807742-8

56 P. 21.

${ }^{57}$ COUCEIRO, A. : o. c., II, p. 426

${ }^{58}$ Se conservan diez ejemplares en Galicia. Citamos tan sólo dos, Santiago (Biblioteca Xeral Universitaria, GA. 693) y Pontevedra (Museo, CA. 604)

${ }^{59}$ FERRERAS, J. I. : o. c., pap. 2048

${ }^{60}$ FERRERAS, J. I.: $o$. c., pap. 1530 
Glorias de Pontevedra, drama histórico en 3 actos, por D. A. J. dos Reis ............................... 4

\section{Reis, Antonio José dos}

Glorias de Pontevedra: drama histórico en 3 actos/ Antonio José dos Reis.

Pontevedra: [s. n.], 1870 (Impr. de José A. Antúnez, Comp.)

$59 \mathrm{pp}$.

CCPB: 000305284-2 $2^{61}$

Gramática gallega, edición de $1868 \ldots \ldots \ldots \ldots \ldots \ldots \ldots \ldots$

Saco y Arce, Juan Antonio. ${ }^{62}$

Gramática gallega / por Juan A. Saco y Arce.

Lugo: [s. n.], 1868, (Imprenta de Soto Freire).

XI, 313 pp.

CCPB: 000171441-463

Historias y cuentos de mi país,

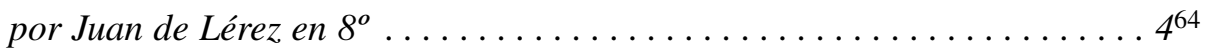

\section{Lérez, Juan de}

Historias y cuentos de mi país/por Juan de Lérez.

Pontevedra: [s. n.], 1864 (Imprenta de Antúnez Vilas y Compañía).

XVII; 157 pp.

CCPB: 0001751114- $\mathrm{X}^{65}$

Juan de Lérez es el pseudónimo de José Benito Amado (1822-1886) ${ }^{66}$

Historia de un homem ........................... 10

No localizada.

${ }^{61}$ Ejemplar editado en la imprenta que anuncia este catálogo y se conserva un único ejemplar en la Biblioteca Pública de esta ciudad, con signatura V.12 (10) con dedicatoria manuscrita del autor. No lo cita Couceiro.

${ }^{62}$ COUCEIRO, A.: $o$. c., III, pp. 267-268.

${ }^{63}$ Se custodian en Galicia 21 ejemplares de esta edición. Citamos tan sólo dos (A Coruña, Diputación, M/912) y Pontevedra (Museo, G-14670, con dedicatoria del autor.)

${ }^{64}$ P. 23

65 Pontevedra, Biblioteca Pública, Fondo “Jesús Muruais” M-2844, y Museo, S-512, V. S. A. 14853 y FV-3573 (1).

${ }^{66}$ COUCEIRO, A.: o. c., I, p. 56 
Historia imparcial de

la Emperatriz Eudoxia . . . . . . . . . . . . . . . . . . . . . . 4

Montegrón, Pedro (1745-1821).

Eudoxia, hija de Belisario: libro primero/ por Pedro Montegrón.

Barcelona: por Juan Ignacio Jordi, 1815.

342 pp.

CCPB: 000457631-4

Libritos del Santo Jubileo . . . . . . . . . . . . . . . 24 rs. unidad 67

Velez, Rafael, Arzobispo de Santiago (1824-1850)

Publicación del Jubileo del Año Santo, é instrucción pastoral que con este motivo da a todos sus fieles/ el... Arzobispo de Santiago, D. Fr. Rafael de Vélez.

[Santiago] en dicha ciudad: [s. n.], 1826, (En la imprenta de D. Juan Francisco Montero).

21 pp.

CCPB: 000166927-368

La señora de Pantalón 14

Kock, Charles-Paul de (1793-1871). ${ }^{69}$

Madame Pantalon/ Ch. Paul de Kock; [avec une gravure par Delannoy d'après Bertall]

Paris: Ferd. Sartorius, éditeur, 1869 (Imp. Simon Raçon et Compagnie).

278, [2] pp., [1] h. de lám.

Serie: Collection illustrée

CCPB: 000716643-5

La familia. Poesías de Sansón

Sansón, José Plácido. ${ }^{70}$

La Familia: Poesías/

$2^{\mathrm{a}}$ ed.

${ }^{67}$ p. 26

68 Ejemplares en Santiago, Instituto Teológico Compostelano (Foll. 70-1), Instituto P. Sarmiento (Foll. 72-16 y Foll. 191-5169), Biblioteca Xeral (13197 (8), 2425, 2426, RSE. Misc. 16(9), Ga. Foll. 14-14 y Ga. Foll. 18-20) y PP. Franciscanos, Biblioteca, (M- 56). En Pontevedra, Museo, (Foll. Gal. $2.5 \mathrm{n}^{\mathrm{o}} 34$ y FVF 27/10).

${ }^{69}$ MONTESINOS, J. F.: $o$. c., pp. 210-211.

${ }^{70}$ FERRERAS, J. I.: $o . c$., pap. 1854 
Madrid: [s. n.], 1864 (Imprenta de M. Tello).

XII, 219, II pp.

CCPB: 000680311-3

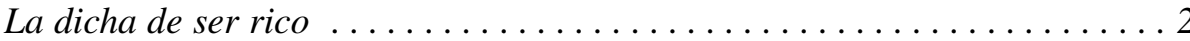

Conscience, Henri. ${ }^{71}$

García Balmaseda, Joaquina, trad.

La dicha de ser rico/ por Henri Conscience; traducida por Joaquina García Balmaseda.

Madrid: [s. n.], 1862 (Imprenta y oficinas de La Correspondencia).

$29 \mathrm{pp}$.

Serie: Biblioteca de La Correspondencia

CCPB: 000629811-7

La resurrección de Rocambole, 2 tomos . . . . . . . . . . . . . . . 28

Ponson du Terrail, Pierre-Alexis, vicomte de (1829-1871). ${ }^{72}$

La résurrection de Rocambole/ Ponson du Terrail.

Éd. illustrée de plus de cent vignettes gravées sur bois/ par Delaville... [et al.] sur dessins de Gerlier.

Paris: Charlieu fréres et Huilery, 1866

420 pp.:il.

CCPB: 00697065-6

La Baronesa de Blaquiskof, 2 tomos . . . . . . . . . . . . . . . . 14

Kock, Charles-Paul de (1793-1871). ${ }^{73}$

Mejía, Rafael, trad.

La Baronesa de Blaguistof/ novela escrita en francés por Ch. Paul de Kock; traducida por Rafael Mejía; ilustrada con una lámina grabada en acero.

Madrid: Carlos Bailly-Bailliére, 1866.

319 pp., [1] h. de lám.

CCPB: 00679068-2

\footnotetext{
71 No lo cita Montesinos.

72 No lo cita Montesinos.

${ }^{73}$ MONTESINOS, J. F.: o. c., pp. 210-213
} 
La mujer de 30 años ............................... 14

Balzac, Honoré de (1799-1850) ${ }^{74}$

Hernández, Enrique, trad.

La mujer de treinta años: escenas de la vida privada/ novela escrita en francés por H. de Balzac; traducida por Enrique Hernández.

Madrid. Carlos Bailly-Bailliére, 1867.

$286 \mathrm{pp}$.

CCPB: 000097599-0

La fiebre de oro ............................... 16

Aimard, Gustave (1818-1883)

Sáenz de Urraca, J. F., trad.

La fiebre de oro: novela escrita en francés/ por M. Gustavo Aimard; traducción de J. F. Sáenz de Urraca.

Madrid: Carlos Bailly-Bailliére, 1867.

349 pp.

Gustave Aimard es el pseudónimo de Olivier Gloux.

CCPB: 00084647-3

Lecturas amenas, por Ochoa ........................ 14

Ochoa, Eugenio de (1815-1872) ${ }^{75}$

Lecturas amenas: sacadas de varios autores extranjeros / por D. Eugenio de Ochoa.

París: E. Tunot y Cía., 1864.

314 pp.

Madrid, Biblioteca Nacional (1/76982)

Los hijos del Amor ............................. 8

Sué, Eugène (1804-1857)

D. J. M. P., trad.

Los Hijos del Amor/ novela escrita en francés por Eugenio Sué; traducida al castellano por D. J. M. P.

Madrid: [s. n., s. a.] (Imprenta de Gabriel Gil).

2 v. (T. I. 199 pp.- T. II. 288 pp.)

${ }^{74}$ MONTESINOS, J. F.: o. c., pp. 160-162

${ }^{75}$ FERRERAS, J. I.: $o$. c., pap. 1480

${ }^{76}$ MONTESINOS, J. F. : o. c., pp. 249-253 
Fecha de publicación, según Palau, 1850.

CCPB: 000680240-0

Los Mosqueteros. El Vizconde de Bragelonne en

cuatro tomos y pasta . . . . . . . . . . . . . . . . . . . . . . 20

Dumas, Alexander (1802-1870). ${ }^{77}$

Balaguer, Victor, trad.

El Vizconde de Bragelona: tercera parte de Los Tres Mosqueteros/ por Alejandro Dumas; traducción de Victor Balaguer.

Barcelona: Imprenta y Librería de la Sra. Vda. e Hijo de Mayol, 1849- 1850.

4 v. (T. I. 475 pp., [1] h. de lám.—- T. II. 627 pp., [1] h. de lám.— T. III. 739 pp., [1] h. de lám.-T. IV. 804 pp., [1] h. de lám.)

Serie: Biblioteca popular continua.

CCPB: 000677434-2

Los dramas desconocidos 4 tomos . . . . . . . . . . . . . . . . . . . . . 12

Soulié, Fréderic (1800-1847).

Los dramas desconocidos / novela escrita en francés por Federico Soulié; traducida al castellano.

Madrid: [s. n.], 1846-1847 (Establecimiento Tipográfico de D. Francisco de Paula Mellado).

2 v. (T. I. 298 pp.-T. II. 293 pp.)

CCPB: 000177440-978

La torre de Londres . . . . . . . . . . . . . . . . . . . . . . . . . $44^{79}$

Ainsworth, W. Harrison (1805-1885). ${ }^{80}$

Viale y Baeza, trad.

La Torre de Londres/ por W. Londres, traducida del inglés por Viale y Baeza.

Barcelona: [s. n.], 1844 (Imprenta de Juan Olivares).

2 v. (T. I. V, 313 pp.-- T. II. 274 pp.)

Serie: Teatro de Autores Ilustres, (XII-XIII)

CCPB: 000459076-7

${ }^{77}$ MONTESINOS, J. F.: o. c., pp. 184-189

78 Hay un ejemplar en la Biblioteca Pública de Pontevedra, procedente del Fondo "Jesús Muruais”, con signatura, M. 2279.

${ }^{79}$ P. 27

${ }^{80}$ MONTESINOS, J. F.: o. c., pp. 154-155 
Los Miserables de Víctor Hugo 10 tomos $\ldots \ldots \ldots \ldots \ldots \ldots \ldots \ldots$

Hugo, Víctor (1802-1885). ${ }^{81}$

Fernández Cuesta, Nemesio, trad.

Los miserables/ por Víctor Hugo; traducción de Nemesio Fernández Cuesta.

Madrid: [s. n.], 1862 (Imprenta de Las Novedades, a cargo de A. Querol y Caparrós).

7 v. (T. I.174 pp. - - T. II. 176 pp.-T. III. 176 pp.- T. IV.156 pp. -T. V.156 pp.-T. VI. 150 pp.- T. VII. 212 pp.)

CCPB: 000229652-7

La montaña mágica ${ }^{82}$ de Scot en $8^{\circ} \ldots \ldots \ldots \ldots \ldots \ldots \ldots$ sin precio

Scott, Walther, Sir (1771-1832). ${ }^{83}$

La maga de la montaña/ novela inédita de Walther Scott.

Madrid: Establecimiento tipográfico de D. Francisco de Paula Mellado, editor, 1844.

$128 \mathrm{pp}$.

CCPB: 000222796- $7^{84}$

Librito discreto de diversos juegos de manos

para diversión en cualquier tertulia

o sarao sin perjuicio

25

Minguet e Yrol, Pablo (1733-1778).

Juegos de manos o sea Arte de hacer diabluras y juegos de prendas...: ilustrado con láminas/ por Pablo Minguet, y aumentado considerablemente en esta nueva edición con infinidad de juegos nuevos, y con láminas intercaladas en el texto. Barcelona: [s. n.], 1847, (Imprenta de Manuel Saurí).

VIII, 189, IX pp.:.il.

CCPB: 000595070-8

La escuela del pueblo 20

Ayguals de Izco, Wenceslao (1801-1873). ${ }^{85}$

\footnotetext{
${ }^{81}$ MONTESINOS, J. F.: o. c., pp. 206-207

82 Título mal copiado que coincide con la novela de Thomas Mann.

${ }^{83}$ MONTESINOS, J. F. : o. c., pp. 239-244

${ }^{84}$ Existe un ejemplar en la Biblioteca Pública de Pontevedra, proveniente del Fondo "Jesús Muruais”, con signatura M. 274.

${ }^{85}$ FERRERAS, J. I.: $o$. c., pap. 184
} 
La escuela del pueblo: páginas de enseñanza universal, seguidas de una recopilación de las obras más selectas que se hayan escrito y escriban en todos los países para perfeccionar el entendimiento humano/ su director Wenceslao Ayguals de Izco. Madrid: [s. n.], 1852-1853 (Imprenta de Ayguals de Izco, Hermanos).

$9 \mathrm{v}$.

17 t. en $9 \mathrm{v}$.

CCPB: 00095268-0

Manual de oraciones, Ribadeneira .................. 4

Ribadeneyra, Pedro de (S. I.) (1527-1611).

358.- Manual de oraciones para el uso y aprovechamiento de la gente devota/ escrito por el P. Pedro Ribadeneyra, de la Compañía de Jesús.

Madrid: [s. n.], 1835 (Imprenta de Don Eusebio Aguado).

439 pp.

CCPB: 000235311-8

Manifiesto del Marqués de Rodil . . . . . . . . . . . . . . . . . . . 6

Rodil, José Ramón, Marqués de (1789-1853).

Manifiesto y causa del Teniente Jeneral [sic] Marqués de Rodil. Documentos importantes a la época contemporánea publicados por él mismo.

Madrid: [s, n.], 1838 (Imprenta de la Viuda de Hernando).

$200 \mathrm{pp}$.

CCPB: 000140940-986

Miscelánea de literatura, viages (sic), y novelas

por Ochoa . . . . . . . . . . . . . . . . . . . . . . . . . . . . . . . 14

Ochoa, Eugenio de(1815-1872). ${ }^{87}$

Miscelánea de literatura, viajes y novelas/ por Eugenio de Ochoa.

Madrid: Carlos Bailly-Bailliére, 1867

VIII, 407 pp.

CCPB: 000158538-X

Manual del viagero (sic) en la Catedral de Santiago . . . . . . . . . . . . . 6 Manual del viajero en la catedral de Santiago: reseña histórica de Galicia, glorias de Galicia, edificios de la ciudad de Santiago...

${ }^{86}$ Hay un ejemplar en A Coruña, Biblioteca del Consulado, S 3 F; 1-10; 330.

${ }^{87}$ FERRERAS, J. I. : o. c., pap. 1460 
Madrid: [s. n.], 1847 (Imprenta y Establecimiento de grabado de D. Baltasar González)

67 pp., [4] h. de lám.

CCPB:00078709-48

Memoria sobre el fomento de la pesca . . . . . . . . . . . . . . . 2

Caamaño y Pardo, Juan José, Conde de Maceda (1761-1819). ${ }^{89}$

Sobre la libertad de pesca y fomento de la marinería: informe a la Junta de la Diputación en Madrid de la Real Sociedad Cantábrica/ por su socio... Conde de Maceda.

Madrid: [s. n.], 1815 (Imprenta Real).

29 pp.

CCPB: 000796115-4

Memoria descriptiva e histórica de la Isla de Tambo

sita en la Provincia de Pontevedra, con el recién

establecido Lazareto hasta el presente, escrita

por D. Félix Álvarez-Inclán, benedictino del

ex-convento de Poyo . . . . . . . . . . . . . . . . . . . . . . . $1^{90}$

No localizada.

Mitología por Caballero ......................... pin precio Fernán Caballero (1796-1877). ${ }^{91}$

La mitología contada a los niños; e historia de los grandes hombres de la Grecia/ por Fernán Caballero; obra ilustrada con 100 grabados.

Barcelona: librería de Juan Bastinos e hijo, 1867.

244 pp.:il.

Fernán Caballero es el pseudónimo de Cecilia Bölh de Faber y Larrea.

Memorias de un médico por Dumas . . . . . . . . . . . . . . . . 8

Dumas, Alexandre (1802-1870). ${ }^{92}$

${ }^{88}$ Se conservan seis ejemplares en Galicia. En A Coruña, Biblioteca de la Diputación, tres (BM/773; BM/3378 y M/1019 “Fondo José Mosquera (O vello dos contos)”. En Pontevedra, un ejemplar en el Museo (FV.11845 (3)). En Vigo, dos en la Fundación Penzol (C-26/15)

${ }^{89}$ COUCEIRO, A.: o. c., I, p. 182

${ }^{90}$ Pp. $28-29$

${ }^{91}$ FERRERAS, J. I.: o. c., pap. 385

92 MONTESINOS, J. F.: o. c., pp. 184-189 
D. R. M. T., trad.

Memorias de un médico/ por A. Dumas; novísima traducción por D. R. M. T.

Madrid: Murcia y Martí, 1870 (Imprenta de la Galería Literaria).

2 v. (T. I. 852 pp., [4] h. de láms. —- T. II. 926 pp., [5] h. de láms.):il.

Serie: Galería Literaria.

CCPB000230660-3:

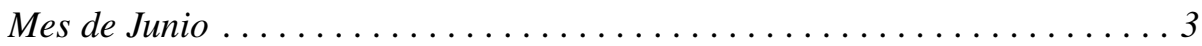

El pequeño mes de junio; El Corazón de Jesús, conocido y amado por los niños/ traducción del francés.

Valencia: [s. n.], 1855 (Imprenta de El Valenciano, antes de Benito Monfort).

CCPB: 000702028-7

También las flores hablan, relieve . . . . . . . . . . . . . . $6^{93}$

Francisco, José Antonio de

También las flores hablan/ por José Antonio de Francisco.

Nueva ed., aum. con el Calendario de las Joyas.

Madrid: Librería de León P. Villaverde, 1861.

$125 \mathrm{pp}$.

CCPB: 000679027-5

Trabajos y miserias de la vida . . . . . . . . . . . . . . . 20

Andueza, José María de (n. 1809). ${ }^{94}$

Trabajos y miserias de la vida: cuadros jocosos-serios/ entretenimiento traducido y original de Aben-Zaide.

Madrid: Boix, 1842.

288 pp., [22] h. de lám.:il.

Aben.-Zaide es el pseudónimo de José María de Andueza.

CCPB: 000075754-3

Vida cristiana o práctica fácil de entablar con

medios y verdades fundamentales contra ignorancias

o descuidos comunes . . . . . . . . . . . . . . . . . . . . . . 1'50

Dutari, Gerónimo (1671-1717) (S. I.).

93 P. 36

${ }^{94}$ FERRERAS, J. I. : o. c., pap. 108 
Vida cristiana ó práctica fácil de entablarla con medios y verdades fundamentales contra ignorancias ó descuidos comunes / por el V. P. Gerónimo [sic] Dutari... Lugo: Soto Freire, editor, 1863 (Imprenta de Soto Freire).

188, [2] pp.

Colección de obras religiosas, $\mathrm{n}^{\circ} 5$

CCPB: 000150927-695

Viage alrededor del mundo, para ilustración de niños . . . . . . . . . D'Urville, Dumont, dir.

Viaje pintoresco alrededor del mundo: resumen general de los viajes y descubrimeintos de Magallanes, Tasman, Dampier.../ publicado en francés bajo la dirección de M. Dumont D’Urville; adornado con mapas..., retratos y seiscientas láminas finas... por M. Saison.

$2^{\mathrm{a}}$ ed.

Barcelona: Imprenta y Librería de Juan Oliveres, 1841.

$3 \mathrm{v}$.

CCPB: 000114976-896

Últimos acontecimientos de Galicia

(reseña histórica de los) en 1846 $4 R S$

Porto, Juan do ${ }^{97}$

Reseña histórica de los últimos acontecimientos políticos de Galicia.../ por Juan do Porto.

La Coruña: [s. n.], 1869, (Imprenta de La Correspondencia de Galicia). 280 pp.

CCPB: 000171667-098

Viage a América de Chateaubriand . . . . . . . . . . . . . . . 8

Chateaubriand, François-René, vicomte de (1768-1848).

Moralejo, José María, trad.

Viage [sic] a la América / por M. de Chateaubriand; traducido por el Doctor Moralejo.

${ }^{95}$ El único ejemplar conservado está en el Instituto Teológico Compostelano, Foll. 113-3.

${ }^{96}$ Museo de Pontevedra, FV. 12006 (I-III)

${ }^{97}$ COUCEIRO, A.: o. c., III, pp. 116-117

98 A Coruña, Consulado, 53 A; 1-3; 114. Anteriormente se había editado esta obra en Madrid, en 1846, en la Imprenta de la Viuda de Burgos. 
París: Librería de Rosa, 1836 (Versalles: Imprenta de Marlin).

3 v. (T. I. 336 pp.- T. II. 264 pp.-- T. III. 197 pp.)

CCPB: 000477168-0

Viages de Chateaubriand a América, Suiza e Italia . . . . . . . . . . . . . 8

Chateaubriand, François-René, vicomte de (1768-1848). ${ }^{99}$

Madina Veytia, Francisco, trad.

Viajes a Italia y América / por F. A. de Chateaubriand; traducción de D. Francisco Madina Veytia.

Madrid: Imprenta de Gaspar y Roig, editores, 1871.

120 pp.:il.

Serie: Biblioteca Ilustrada Gaspar Y Roig.

CCPB: 000152181-0

Vida de Tailleirand .......................

Bastide, Jean- Louis. ${ }^{100}$

R. M. F. P., trad.

Vida relijiosa [sic] y politica de Talleyrand-Périgord, Príncipe de Benevento: desde su nacimiento hasta su muerte; traducida del francés por R. M. F. P.

Barcelona: [s.n.], 1838, (En la imprenta de Tomás Gaspar).

476 p., [3] h. de láms.:il.

CCPB: 000081050-9

Talleyrand-Périgord, Charles Maurice de (1754-1838)

Viageros y bañistas . ....................... 4

Viajeros y Bañistas: espediciones [sic] humorísticas por mar y tierra, chapuzo, lavatorios y lo que colea.

Madrid: [s. n.], 1866 (Imprenta de R. Labajos).

189 pp.

Colección Biblioteca Nacional, $\mathrm{n}^{\mathrm{o}} 4$

CCPB: 000533100-5

A continuación van los títulos “Impresos Generales"101 y "Para copleros"102.

${ }^{99}$ MONTESINOS, J. F.: o. c., pp. 171-175

100 No lo cita Montesinos.

101 Pp. 38-39

102 Pp. 39-40 
Dentro de este último epígrafe reseñamos como curiosas, las siguientes:

"Tablas para el noble juego de las Damas.

Id. para el de la Oca.

Estampas del verdadero San Benito

Saludable remedio contra la peste: cruz de San Zacarías

Rescriptos contra maleficios

Cruces contra las brujas: llamadas rescriptos contra toda clase de enfermedades o maleficios.

Madrid: [s. n., s. a.], (Imprenta Universal).

[2] pp.:il.

La imprenta Universal trabaja ca. 1866-1870

CCPB:000321979-8

Gritos y lamentos de las benditas ánimas del purgatorio

Silvos del Pastor Divino

Silvos del Divino Pastor: canciones que se cantan en las Misiones de los Padres Misioneros.

Pontevedra: [s. n.], 1857 (Imprenta de la Viuda e Hijos de Verea).

$27 \mathrm{pp}$.

CCPB:00176917-0 $0^{103}$

Suspiros de una arrepentida con dos láminas

Romance contra las llamadas sabias que hechan cartas

Nuestra Señora de Valdegimena, abogada de las horas menguadas

Nuestra Señora de Valdegimena, abogada de las horas menguadas, aires corruptos, y mordeduras de perros rabiosos. Alabanzas a la Santísima Virgen que con el título de Nuestra Señora de Valdegimena, se venera en su santuario de la provincia de Ávila.

Madrid: Despacho, Juanelo [s. a.].

[2] pp.:il.

CCPB: 000323017-1

Verídica relación de los desastres causados por huracanes en Calcuta y sobre el Ganges

No localizada

${ }^{103}$ Museo de Pontevedra, Fondo Casal, XXXIV 
Curiosos pormenores aplicados contra los malos vicios y palabras obscenas No localizada

Romance de la guerra franco-prusiana"

\section{Martínez Aparico, Ramiro}

A Francia por un hulano: pasillo original en un acto y en verso escrito a propósito de la guerra franco-prusiana / por Ramiro Martínez Aparicio.

Madrid: [s. n.], 1870 (Imprenta de M. Minuesa).

23 pp.

Estrenado en el Teatro Novedades en la noche del 9 de octubre de 1870.

CCPB: 0003955005-0.

\section{BIBLIOGRAFÍA.}

Catálogo colectivo del Patrimonio Bibliográfico de España (on-line)

ODRIOZOLA, A.: Las imprentas en Pontevedra en el siglo XIX, Pontevedra: Diputación Provincial, 1989.

COUCEIRO FREIJOMIL, A.: Diccionario Bio-bibibliográfico de escritores, Santiago de Compostela: Editorial de los Bibliófilos Gallegos, 1952-1953, 3 v.

MARTÍNEZ, Jesús A.(DIR.): Historia de la edición en España (1836-1936), Madrid: Marcial Pons, 2001.

BAASNER, Frank- ACERO YUS, F. (DiRs.): Doscientos críticos literarios en la España del S. XIX, Madrid: CSIC, 2007.

CABANO VÁZQUEZ, José Ignacio (DiR.): Catálogo colectivo do Patrimonio Bibliográfico de Galicia. A imprenta en Galicia. Século XIX. Santiago de Compostela: Consellería de Cultura, Comunicación social e Turismo, 2002, 2 v.

MONTESINOS, José F.: Introducción a una historia de la novela en España en el Siglo XIX. Seguida del esbozo de una bibliografía española de traducciones de novelas (1800-1850), Valencia: Editorial Castalia, 1972.

FERRERAS, Juan Ignacio: Catálogo de novelas y novelistas españoles del siglo XIX, Madrid: Cátedra, 1979. 